Research Article

\title{
Comparison of Nasal Colonization of Methicillin-Resistant Staphylococcus aureus in HIV-Infected and Non-HIV Patients Attending the National Public Health Laboratory of Central Nepal
}

\author{
Kalash Neupane, ${ }^{1}$ Binod Rayamajhee ${ }^{D},{ }^{2,3}$ Jyoti Acharya, ${ }^{4}$ Nisha Rijal, ${ }^{4}$ \\ Dipendra Shrestha, ${ }^{2}$ Binod G C $\mathbb{D}^{2,3}$ Mahesh Raj Pant, ${ }^{5}$ and Pradeep Kumar Shah ${ }^{1}$ \\ ${ }^{1}$ Department of Microbiology, Trichandra Multiple Campus, Tribhuvan University, Kathmandu, Nepal \\ ${ }^{2}$ National College, Tribhuvan University, Khusibu, Kathmandu, Nepal \\ ${ }^{3}$ Department of Infectious Diseases and Immunology, Kathmandu Research Institute for Biological Sciences, Lalitpur, Nepal \\ ${ }^{4}$ Department of Bacteriology, National Public Health Laboratory, Teku, Kathmandu, Nepal \\ ${ }^{5}$ Department of Microbiology, Kathmandu College of Science and Technology, Kamalpokhari, Kathmandu, Nepal
}

Correspondence should be addressed to Binod Rayamajhee; rayamajheebinod@gmail.com

Received 29 August 2018; Accepted 15 November 2018; Published 4 December 2018

Academic Editor: Lucia Lopalco

Copyright ( $) 2018$ Kalash Neupane et al. This is an open access article distributed under the Creative Commons Attribution License, which permits unrestricted use, distribution, and reproduction in any medium, provided the original work is properly cited.

\begin{abstract}
Background. Staphylococcus aureus is a cardinal source of community- and hospital-acquired infection. HIV infection is a well-recognized risk factor for methicillin-resistant S. aureus (MRSA) carriage and infection. Intrinsically developed antibiotic resistance has sharply increased the burden of MRSA which is often associated with morbidity and mortality of the patients. Moreover, nasal carriage of $S$. aureus plays a significant role in spread of community-associated (CA) S. aureus infections. Methods. This study was conducted from June 2016 to December 2016 at National Public Health Laboratory (NPHL), Kathmandu, with an aim to assess the rate of $S$. aureus nasal carriage and MRSA carriage among HIV-infected and non-HIV patients. A total of 600 nonrepeated nasal swabs were analyzed following standard microbiological procedures, where 300 swabs were from HIV-infected patients while remaining 300 were from non-HIV patients. The isolates were identified on the basis of colony characteristics and a series of biochemical tests. The antibiotic susceptibility test (AST) was performed by the modified Kirby-Bauer disc diffusion method. Inducible clindamycin resistance in isolates was confirmed by the D-test method. Results. Overall, out of 600 nasal swabs of patients tested, 125 (20.8\%) were S. aureus nasal carriers which included 80 out of $300(26.66 \%)$ among HIV-infected patients and 45 (15\%) out of 300 among non-HIV patients, and the result was statistically significant $(p=0.0043)$. Among the isolated S. aureus, $11(13.8 \%)$ MRSA were confirmed in HIVinfected while $3(6.7 \%)$ MRSA were detected from non-HIV patients. A higher number of $S$. aureus carriers was detected among HIV-infected males 40 (26.49\%), whereas MRSA carriage was more prevalent among HIV-infected females 7 (5.1\%). Among the HIV-infected, patients of age group 31-40 years were the ones with highest carriage rate 36 (45\%), while in nonHIV patients, the highest rate $13(28.9 \%)$ of carriage was detected among the patients of age group 21-30 years. Statistically significant difference was found between S. aureus carriage and HIV infection in patients $(p<0.05)$. Higher rate $2 / 3(66.7 \%)$ of inducible clindamycin resistance in MRSA was detected from non-HIV patients in comparison to HIV-infected patients $7 / 11(63.63 \%)$ while the result was statistically insignificant $(p>0.05)$. All the MRSA isolates $(100 \%)$ were resistant against co-trimoxazole while ciprofloxacin showed high rate of sensitivity towards both MSSA and MRSA. None of the isolates were detected as VRSA. The major factors associated with nasal colonization of $S$. aureus were close personal contact, current smoking habit, and working or living in a farm $(p<0.05)$. Conclusion. Regular surveillance and monitoring of MRSA nasal carriage and antibiotic susceptibility pattern are of prime importance in controlling $S$. aureus infections especially in high risk groups like HIV-infected patients.
\end{abstract}




\section{Introduction}

Staphylococcus aureus is the most prevalent pathogen in community and health care setting and has been a serious threat to human health since its discovery [1]. S. aureus, especially methicillin-resistant $S$. aureus (MRSA), is responsible for varieties of maladies ranging from folliculitis to food poisoning. Moreover, it is responsible for causing life-threatening infections such as endocarditis, necrotizing pneumonitis, and osteomyelitis, among others [2]. Community-acquired MRSA (CA-MRSA) strain has emerged as a challenging pathogen which is frequently isolated from military personnel, drug users, athletes, and men who have sex with men [3]. The enduring threat and changing nature of $S$. aureus as a leading infectious pathogen has been well reported [4]. It is a common human skin colonizer, and pathogen armed with various classes of virulence factors including pore-forming toxins, superantigens, phagocytosis inhibitors, and biofilm forming capacity [5].

Some of the most severe infections caused by $S$. aureus include bacteremia, pneumonia, osteomyelitis, toxic shock syndrome, acute endocarditis, myocarditis, meningitis and abscesses in muscles, genitourinary tract infection, infection of central nervous system and various intraabdominal organs [6]. Methicillin-resistant S. aureus (MRSA) is a predominant cause of infection both in clinical and community settings which has amplified the disease burden to a major public health problem [7]. Colonizing feature of S. aureus is a potential factor for infection, an individual colonized with MRSA strain has a two to twelve fold more risk of subsequent infection [8]. Colonization and infection of MRSA has been recognized more in HIV-infected persons [9]. Moreover, higher prevalence of pathogenic MRSA strains have been documented among HIV-infected pediatric patients [10]. HIV infection and young age are considered as independent risk factors of MRSA infection [1]. High nasal carriage rate of MRSA in HIV infected persons may require early interventions. Hospitalized HIV-infected patients are nearly 17 times more likely to get $S$. aureus infection in comparison to non-HIV patients [11]. Not only this, the increasing prevalence of infections caused by MRSA has been difficult to treat due to the high rate of resistance to commonly prescribed antibiotics [12]. Determination of $S$. aureus nasal carriage rate and antibiotic resistance profiles along with molecular typing of nasal $S$. aureus isolates in healthy populations is necessary to identify risk factors associated with $S$. aureus infection [13-15].

In Nepal, reports on nasal carriage rate in vulnerable groups like HIV sero-positive individuals are very limited. Therefore, this study was carried out to determine the rate of nasal carriage of $S$. aureus in HIV-infected patients which was compared with healthy carriers. Furthermore, the antibiotic resistance patterns of isolates to commonly prescribed antibiotics, was also investigated. This study could be beneficial in management of MRSA infections, particularly in HIV-infected patients, while suggesting appropriate measures for controlling the possible risks of $S$. aureus infections in HIV-infected patients.

\section{Methods and Materials}

2.1. Study Design. This study was a prospective crosssectional study. The study was carried out at bacteriology laboratory of National Public Health Laboratory, Teku, Kathmandu. Study population consisted of HIV-infected and non-HIV infected patients from whom nasal swab was collected. A total of 600 nasal swabs were collected for laboratory diagnosis, of which 300 swabs each were from HIV and nonHIV patients. The study duration was 6 months from June 2016 to December 2016. Preformed questionnaire was used to obtain the clinical history and demographic information of each patient. We also collected the information about health conditions and environmental factors of the subjects which were hypothesized to be major factors associated with MRSA colonization in the study population.

2.2. Inclusion and Exclusion Criteria. Patients with known history of HIV infection (seropositive) and noninfection (seronegative) were included in the study. Nasal swabs collected with standard operating procedure were accepted where complete label and strict sterile condition was maintained. Those samples that were improperly labeled and those from the patients who were non-HIV but having other immunodeficiency conditions like renal transplant, cancer, diabetes, liver cirrhosis, malignancy, chronic cardiovascular diseases, or consuming any immunosuppressant medicine were excluded from the study. Additionally, patients who had received any kind of antibiotics within the previous two weeks were also excluded from this study.

2.3. Specimen Collection and Transport. Sampling procedure was done by a well-trained laboratory technician. Nasal swabs were collected by using sterile cotton swabs moistened with sterile normal saline. Each nasal swab was obtained by rotating 2-3 times in the anterior nares of patients' and was transported to bacteriology department quickly for further processing [16]. All the collected swabs were transported in a zip lock bag to the laboratory. No transport medium was used because the bacteriology department and sample collection sites were adjacent to each other and additionally, drying of swab was prevented.

\subsection{Laboratory Analysis}

2.4.1. Microscopic Observation. The nasal swabs were evenly smeared on clean, dry, and grease-free glass slide. Then smear was heat fixed and stained by Gram stain procedure [17]. The stained smear was observed microscopically using the 100x objective for presence of Gram positive cocci in grape-like clusters.

2.4.2. Culture of Nasal Swabs. Primary culture was done on $5 \%$ sheep blood agar, Mac-Conkey agar, and Mannitol salt agar under aseptic conditions. Identification of isolated colonies was done by colony morphology, Gram staining, catalase test, slide coagulase test, and tube coagulase test [18]. 
2.4.3. Antibiotic Susceptibility Testing. Antibiotic susceptibility test was performed on Mueller Hinton Agar (MHA) by modified Kirby-Bauer disk diffusion method as per the Clinical Laboratory and Standards Institute 2016 guidelines [19] and the obtained results were interpreted accordingly. The antibiotics discs and concentrations used were ciprofloxacin $(5 \mu \mathrm{g})$, penicillin $\mathrm{G}(30 \mu \mathrm{g})$, gentamicin $(30 \mu \mathrm{g})$ cotrimoxazole $(25 \mu \mathrm{g})$, cefoxitin $(30 \mu \mathrm{g})$, erythromycin $(30 \mu \mathrm{g})$, tetracycline $(30 \mu \mathrm{g})$, clindamycin $(2 \mu \mathrm{g})$, and vancomycin $(30 \mu \mathrm{g})$. The diameter of zone of inhibition (ZOI) was measured and the results were interpreted. Antimicrobials doses were selected on the basis of prescription frequency by physician. Minimum inhibitory concentration (MIC) values of used antibiotics were unable to determine due to unavailability of all antibiotics powder at the time of study period.

2.4.4. Screening of Methicillin-Resistant S. aureus (MRSA). Screening for methicillin resistance in S. aureus was done by using cefoxitin $(30 \mu \mathrm{g})$ antibiotic disc following modified Kirby-Bauer disc diffusion technique. Those $S$. aureus that showed zone size of $\leq 21 \mathrm{~mm}$ around cefoxitin disk were confirmed as MRSA strain.

\subsubsection{Screening of Inducible Clindamycin-Resistant S. aureus.} The macrolide-inducible clindamycin resistance in S. aureus was detected by $\mathrm{D}$ test method [20]. 0.5 McFarland standard bacterial suspensions of the test isolate was inoculated on MHA plate and then clindamycin $(2 \mu \mathrm{g})$ and erythromycin $(15 \mu \mathrm{g})$ discs were kept $15 \mathrm{~mm}$ edge to edge on the same plate. Then the plates were aerobically incubated overnight at $37^{\circ} \mathrm{C}$. After the incubation period, flattening of zone (Dshaped) around the clindamycin disc was considered as inducible clindamycin resistance positive in S. aureus. Results of erythromycin-resistant $S$. aureus after D-test were interpreted into three phenotypic categories; $\mathrm{MS}_{\mathrm{B}}$ (macrolide-streptogramin $\mathrm{B}$ ), inducible $\mathrm{MLS}_{\mathrm{B}}\left(\mathrm{MLS}_{\mathrm{B}}\right)$, and constitutive ${ }_{\mathrm{C}} \mathrm{MLS} \mathrm{S}_{\mathrm{B}}$ phenotype.

2.5. Quality Control. Media preparation, inoculation, and culture were performed in strict aseptic conditions. The prepared media were checked for the appearance of pure growth of organisms by using ATCC control strain. Stable strain of S. aureus ATCC 25923 was used as the control organism for the laboratory procedures. The thickness of MHA plates was maintained at $4 \mathrm{~mm}$ and the $\mathrm{pH}$ at 7.2-7.4. Microscope, incubator, centrifuge, refrigerator, water bath, autoclave, and hot air oven were checked regularly to ensure the correct functioning of equipment for the reliability of results. The temperature for all equipment was monitored and recorded.

2.6. Data Analysis. All data were analyzed using SPSS version 21.0. Chi square test was calculated where $p$ value of $<0.05$ was considered statistically significant at $95 \%$ of confidence level.

\section{Results}

Among 600 nasal swabs investigated for determination of $S$. aureus and MRSA nasal carriage, 125 patients were found to be colonized with $S$. aureus among which 80 specimens were from HIV-infected and 45 from non-HIV individuals. The total prevalence of $S$. aureus colonization in HIV was found to be $26.66 \%$ while colonization in non-HIV was $15 \%$. The MRSA colonization among the HIV patients was $13.8 \%$ (11/80) among $80 \mathrm{~S}$. aureus isolates, whereas the MRSA colonization in non-HIV was $6.7 \%(3 / 45)$ among $45 \mathrm{~S}$. aureus isolates.

Out of 300 nasal swabs analyzed from HIV-infected individuals, $S$. aureus isolates were 80 , of which $26.5 \%$ (40/151) were male carriers whereas $23.2 \%$ (32/138) were female carriers and among third gender $72.7 \%(8 / 11)$ were S. aureus carriers (Table 1). Similarly, out of $45 \mathrm{~S}$. aureus isolates from the 300 nasal swabs collected from non-HIV patients; $17.5 \%$ (27/154) were isolated from males whereas $12.3 \%(18 / 146)$ were from females. The study reveals that the $S$. aureus carrier percentage was higher among males in HIV-infected as well as in non-HIV patients (Table 2). The nasal carriage rate was not statistically significant with gender of patients $(p>0.05)$.

In HIV-infected patients, a high rate of $S$. aureus nasal carriage was found among the patients of age group 31-40 years with 36 (45\%) followed by $21-30$ years with 15 (18.8\%), 41-50 years with 13 (17.5\%), 11-20 years with 8 (10\%) whereas $S$. aureus was not detected from the patients of age group 61 year or above (Table 1). Similarly, in non-HIV patients the high rate of $S$. aureus nasal carriage was found among the patients of age group 31-40 years with 12 (26.7\%) followed by 11 (24.4\%) in age group 41-50 years, 7 (15.6\%) in age group 51-60 years, $5(11.1 \%)$ in patients of age group 61 year or above whereas $S$. aureus was not detected from the patients of age group 10 years or less (Table 2). S. aureus carrier rate was statistically insignificant in relation to age groups of patients $(p>0.05)$.

Among the $300 \mathrm{HIV}$-infected patients, the growth of $S$. aureus was found in 80 (26.6\%), of which 11 (13.8\%) were confirmed as MRSA strains and remaining 69 (86.2\%) were methicillin-sensitive Staphylococcus aureus (MSSA) (Table 1). On the other hand, from the 300 non-HIV patients 45 (15\%) $S$. aureus were isolated where $3(6.7 \%)$ isolates were confirmed as MRSA strain whereas $42(93.3 \%)$ were MSSA strains (Table 2). The results claim higher percentage of MRSA among HIV-infected patients as compared to non-HIV patients. The association between MRSA isolates and HIVinfected patients was found to be statistically significant $(p<0.05)$. More MRSA isolates were isolated from HIVinfected male patients $6(7.5 \%)$ than from females $4(5 \%)$ whereas $1(1.3 \%)$ was isolated from third gender patients with known HIV infection. MSSA was found higher 34 (42.5\%) in male patients as compared to female patients $28(35 \%)$. Similarly, more MRSA 2 (4.4\%) was found among male nonHIV patients whereas one MRSA was reported from female patients. MSSA isolates rate was also higher among male 25 (55.6\%) as compared to female $17(37.8 \%)$ patients (Figure 1). MRSA and MSSA carrier rate was statistically insignificant in relation with gender of patients $(p>0.05)$. 
TABLE 1: Socio demographic characteristics of the HIV-infected patients and ratio of S. aureus carriage.

\begin{tabular}{|c|c|c|c|c|c|}
\hline Demographic features & Type of patients & S. aureus (no. (\%)) & MRSA (no. (\%)) & No growth & Total (no. (\%)) \\
\hline Gender & HIV-infected & & & & \\
\hline Male & 151 & $40(50)$ & $6(7.5)$ & $111(50.4)$ & $151(50.3)$ \\
\hline Female & 138 & $32(40)$ & $4(5)$ & $106(48.2)$ & $138(46)$ \\
\hline Third gender & 11 & $8(10)$ & $1(1.3)$ & $3(1.4)$ & $11(3.7)$ \\
\hline Total & 300 & $80(26.7)$ & $11(13.8)$ & $220(73.3)$ & $300(100)$ \\
\hline \multicolumn{6}{|l|}{ Age in years } \\
\hline$\leq 10$ & 18 & $4(5)$ & 0 & $14(77.8)$ & $18(6)$ \\
\hline $11-20$ & 29 & $8(10)$ & $2(2.5)$ & $21(72.4)$ & $29(9.7)$ \\
\hline $21-30$ & 59 & $15(18.8)$ & $3(3.8)$ & $44(74.6)$ & $59(19.7)$ \\
\hline $31-40$ & 93 & $36(45)$ & $5(6.3)$ & $57(61.3)$ & $93(31)$ \\
\hline $41-50$ & 76 & $13(17.5)$ & $1(1.3)$ & $63(82.9)$ & $76(25.3)$ \\
\hline $51-60$ & 21 & $4(5)$ & 0 & $17(80.9)$ & $21(7)$ \\
\hline$\geq 61$ & 4 & 0 & 0 & $4(100)$ & $4(1.3)$ \\
\hline Total & 300 & $80(26.7)$ & $11(13.8)$ & $220(73.3)$ & $300(100)$ \\
\hline
\end{tabular}

TABLE 2: Socio demographic characteristics of the non-HIV patients and ratio of S. aureus carriage.

\begin{tabular}{|c|c|c|c|c|c|}
\hline Demographic features & Type of patients & S. aureus (no. (\%)) & MRSA (no. (\%)) & No growth & Total (no. (\%)) \\
\hline Gender & Non-HIV patients & & & & \\
\hline Male & 154 & $27(60)$ & $2(4.4)$ & $127(49.8)$ & $154(51.3)$ \\
\hline Female & 146 & $18(40)$ & $1(2.3)$ & $128(50.2)$ & $146(48.7)$ \\
\hline Third gender & 0 & 0 & 0 & 0 & 0 \\
\hline Total & 300 & $45(15)$ & $3(6.7)$ & $255(85)$ & $300(100)$ \\
\hline \multicolumn{6}{|l|}{ Age in years } \\
\hline$\leq 10$ & 16 & 0 & 0 & $16(100)$ & $16(5.3)$ \\
\hline $11-20$ & 33 & $4(8.9)$ & 0 & $29(87.9)$ & $33(11)$ \\
\hline $21-30$ & 45 & $6(13.3)$ & 0 & $39(86.7)$ & $45(15)$ \\
\hline $31-40$ & 101 & $12(26.7)$ & $2(4.4)$ & $89(88.1)$ & $101(33.7)$ \\
\hline $41-50$ & 66 & $11(24.4)$ & $1(2.2)$ & $55(83.3)$ & $66(22)$ \\
\hline $51-60$ & 26 & $7(15.6)$ & 0 & $19(73.1)$ & $26(8.7)$ \\
\hline$\geq 61$ & 13 & $5(11.1)$ & 0 & $8(61.5)$ & $13(4.3)$ \\
\hline Total & 300 & $45(15)$ & $3(6.7)$ & $255(85)$ & $300(100)$ \\
\hline
\end{tabular}

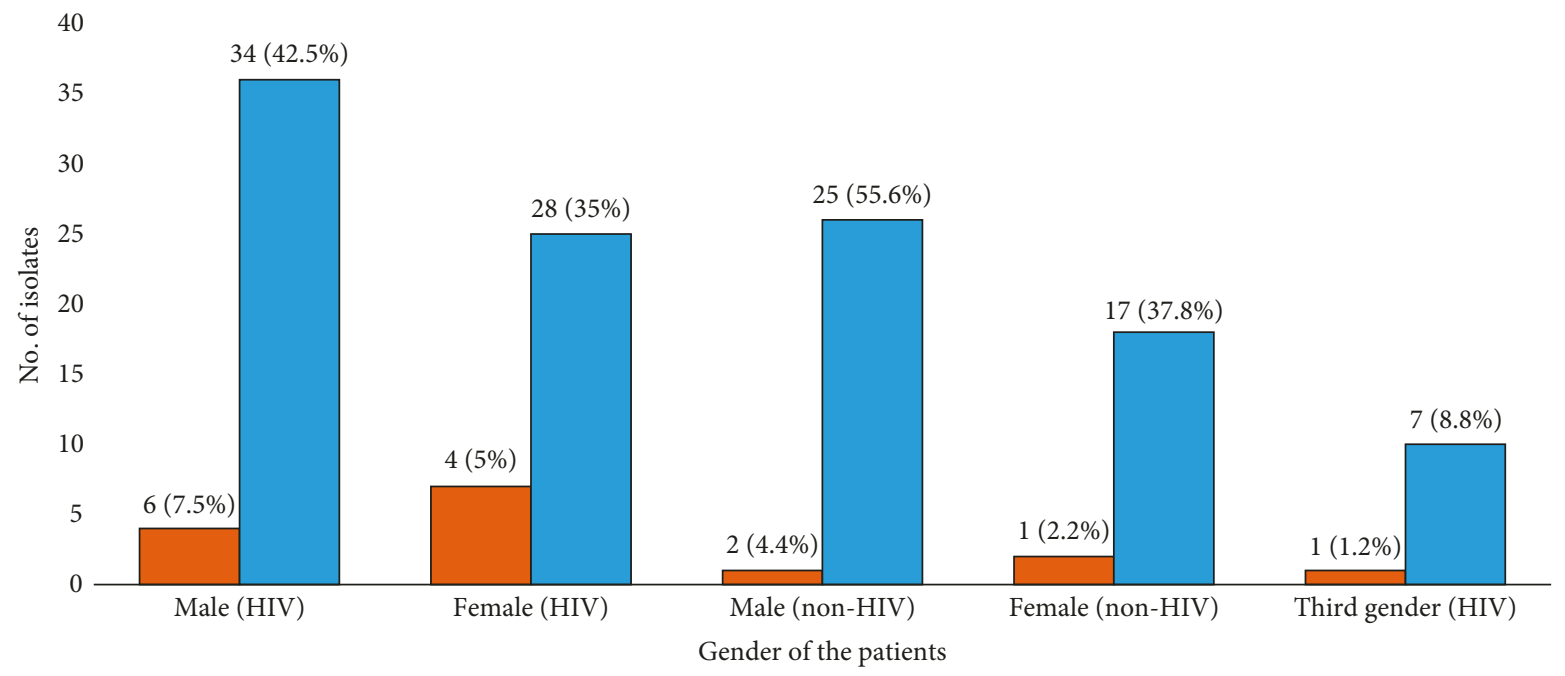

MRSA

MSSA

Figure 1: Gender-wise distribution of MRSA and MSSA isolates among HIV-infected and non-HIV patients. 
Among the $S$. aureus isolates, the highest rate of antibiotic susceptibility was observed against vancomycin (100\%) followed by tetracycline $(94.4 \%)$, cefoxitin $(88.8 \%)$ and gentamycin (80.8). High rate of resistance was seen towards erythromycin (41.6\%) followed by co-trimoxazole (40.8\%). Most 10 (71.4\%) of the MRSA were resistant towards ciprofloxacin (Table 3).

Among $125 \mathrm{~S}$. aureus isolates from HIV-infected and non-HIV patients, percentage of both inducible and constitutive clindamycin resistance was higher in MRSA as compare to MSSA strains. Inducible $\mathrm{MLS}_{\mathrm{B}}-\left(\mathrm{iMLS}_{\mathrm{B}}\right)$ resistance, constitutive $\mathrm{MLS}_{\mathrm{B}}$ resistance, and macrolidestreptogramin $\mathrm{B}\left(\mathrm{MS}_{\mathrm{B}}\right)$ resistance were detected in 30 (24\%), $19(15.2 \%)$, and $34(27.2 \%)$ isolates of $S$. aureus, respectively. Higher rate of $\mathrm{iMLS}_{\mathrm{B}}$ and $\mathrm{CMLS}_{\mathrm{B}}$ was seen among $S$. aureus isolated from $\mathrm{HIV}$-infected patients while the rate of inducible $M_{\mathrm{BLS}}$ resistance was statistically insignificant as to the origin of $S$. aureus isolates i.e. HIV-infected and nonHIV patients $(p>0.05)$ (Table 4$)$.

Nasal colonization rate of $S$. aureus was found significantly higher among the patients who used to work or live on a farm $(p<0.05)$. Similarly, inpatients showed higher rate 69 (55.2\%) of $S$. aureus carriage as compared to outpatients 56 (44.8\%), which was statistically significant data $(p=0.005)$. Additionally, patients with current smoking habit showed higher rate $76(60.8 \%)$ of $S$. aureus carriage as compare to non-smokers $49(39.2 \%)(p=0.0051)$. Patients who had a job with close human contact were more prone to being a $S$. aureus carrier $89(71.2 \%)$ as compared to those not having close human contact jobs $36(28.8 \%)(p=0.0076)$ (Table 5).

\section{Discussion}

Colonization of MRSA significantly increases the risk of $S$. aureus infection during hospital stay and even after discharge [21]. Furthermore, nasal carriage of S. aureus is a cardinal source of infection and nasopharynx is considered as the main reservoir of $S$. aureus [1]. The outbreaks of MRSA infection especially in group of people like security shelters, health care centers and refugee camps strongly supports the theory that the spread of $S$. aureus requires close personal contact [22]. The rate of nasal carriage depends on various factors like clinical environment, geographical area, occupation of patients, and patient's immune status among others. Previous studies have reported up to $33 \%$ nasal colonization of S. aureus among HIV-infected population [12].

Total of 600 nasal swabs were analyzed in this study where 300 nasal swabs were from HIV-infected patients and remaining 300 were from non-HIV patients. The prevalence of $S$. aureus in HIV-infected patients was found to be more than the rate in non-HIV patients. This study shows the total prevalence rate of nasal carriage of $S$. aureus to be $20.8 \%$ (125/600). HIV-infected patients have higher rate of $S$. aureus nasal carriage than non-HIV infected patients and the result was statistically significant $(p<0.05)$. High rate of $S$. aureus colonization in HIV infected individuals can lead to severe infections in this group of people [23]. Immune status along with an individual behavioral features can play a positive role in $S$. aureus colonization and subsequent infection [24]. In a similar study carried out by Gonsu et al. (2013) in Cameroon, $40.6 \%$ of $S$. aureus nasal carriage was reported, whereas $34.6 \%$ of isolated S. aureus were MRSA among medical staffs and adult hospitalized patients [25]. Lilian et al. (2013) from Brazil have published a report of $27.2 \%$ as the prevalence of nasal colonization with $S$. aureus in patients with HIV/AIDS whereas the rate of MRSA was $21.8 \%$ [26]. Furthermore, the study has reported that nasal colonization of $S$. aureus was found to be more among the patients with CD4 cell count $<200$ cells $/ \mathrm{mm}^{3}$ and who had a high HIV viral load. Hence, HIV-infected patients are more vulnerable group to develop infections due to MRSA. On the other hand, $S$. aureus nasal colonization rate was found $18.4 \%$ among the healthy secondary school going students in Iraq whereas only $2.04 \%$ isolates were found to be MRSA [27].

The nasal carriage rate of $S$. aureus can be directly linked with underlying diseases, surrounding environment, age of patients and close contact activities [28]. In a study conducted among health care workers of Pakistan, 18.2\% were nasal carriers of $S$. aureus where the count was lead my midwives $(30 \%)$ then maintenance staffs of the hospital (28.6\%) where only $1.5 \%$ of isolates were MRSA [29]. The real reason behind higher rate of $S$. aureus nasal colonization in midwives is unclear but this could be due to frequent contact with hospitalized patients and longer stay in hospital environment [30]. In a result reported by Khanal et al. (2015) the highest rate of MRSA nasal carriage was noted among hospital nurses which was $7.8 \%$ followed by hospital staffs of surgical wards and operating department whereas high number of $S$. aureus isolates $(20.8 \%)$ were reported from doctors [31].

This study reveals higher rate of $S$. aureus colonization among patients of age group 31-40 years both in HIVinfected and no-HIV patients with prevalence rate $45 \%$ and $26.7 \%$, respectively. Similarly, in a study carried out by Reinato et al., they have reported $36.1 \%$ of S. aureus colonization rate among the HIV/AIDS patients of age group 30-39 years [26]. The risk factors that have been associated with the emergence of MRSA infections among HIV infected individuals are not practically understood well but may be due to some possible risk factors, such as living conditions, prior hospitalization, use of fluoroquinolones and third generation cephalosporin antibiotics, intravenous drug usage, other secondary coinfections and low level of CD4 cells [32]. Some studies have suggested that participation in high risk sexual behaviors, use of public bath, anal intercourse, men who have sex with men (MSM), and sex with multiple partners may flourish the transmission of $S$. aureus and MRSA [33-35].

In this study, more $S$. aureus isolates were reported from males as compared to females both in HIV-infected and nonHIV patients though the result was not statistically significant $(p<0.05)$. The nasal carriage of $S$. aureus is found to be higher among male of active age groups who are mostly involved in outdoor activities, frequently visit to health care centers, and regular exposure to mass of people [31]. In contrast, higher rate of $S$. aureus nasal colonization was reported in female 
TABLE 3: Antibiotic susceptibility pattern of MSSA and MRSA bacteria isolated from HIV-infected and non-HIV patients.

\begin{tabular}{|c|c|c|c|c|c|c|c|c|c|c|}
\hline \multirow{2}{*}{ Isolates } & \multirow{2}{*}{$\begin{array}{l}\text { Type of } \\
\text { patients }\end{array}$} & \multirow{2}{*}{ RXN } & \multicolumn{8}{|c|}{ Antimicrobial agents (no. (\%)) } \\
\hline & & & $\mathrm{CX}$ & CIP & COT & GEN & VA & $\mathrm{P}$ & $\mathrm{E}$ & $\mathrm{TE}$ \\
\hline \multirow{2}{*}{$\operatorname{MSSA}(n=69)$} & \multirow{2}{*}{ HIV-infected } & S & $69(100)$ & $56(81.2)$ & $45(65.2)$ & $60(86.9)$ & $69(100)$ & $57(82.6)$ & $41(59.4)$ & $66(95.7)$ \\
\hline & & $\mathrm{R}$ & 0 & 13( & $24(34$ & $9(1$ & 0 & $12(1$ & $28(4$ & $3(4.3)$ \\
\hline \multirow{2}{*}{$\operatorname{MSSA}(n=42)$} & \multirow{2}{*}{ Non-HIV } & S & $42(100)$ & $33(78.6)$ & $24(57.1)$ & $36(85.7)$ & $42(100)$ & $39(92.38$ & $27(64.3)$ & $42(100)$ \\
\hline & & $\mathrm{R}$ & 0 & $9(21.4)$ & $18(42.9)$ & $6(14.3)$ & 0 & $3(7.2)$ & $15(35.7)$ & 0 \\
\hline \multirow{2}{*}{$\operatorname{MRSA}(n=11)$} & \multirow{2}{*}{ HIV-infected } & S & 0 & $3(27.3)$ & $5(45.5)$ & $3(27$. & $11(100)$ & 0 & $4(36.4)$ & $8(72.7)$ \\
\hline & & $\mathrm{R}$ & $11(100)$ & $8(72.7)$ & $6(54.5)$ & $8(72.7)$ & 0 & $11(100)$ & $7(63.6)$ & $3(27.3)$ \\
\hline \multirow{2}{*}{$\operatorname{MRSA}(n=3)$} & \multirow{2}{*}{ Non-HIV } & S & 0 & $1(33.3)$ & 0 & $2(66.7)$ & $3(100)$ & 0 & $1(33.3)$ & $2(66.7)$ \\
\hline & & $\mathrm{R}$ & $3(100)$ & $2(66.7)$ & $3(100)$ & $1(33.3)$ & 0 & $3(100)$ & $2(66.7)$ & $1(33.3)$ \\
\hline \multirow{2}{*}{ Total $(n=125)$} & & S & $111(88.8)$ & $93(74.4)$ & $74(59.2)$ & $101(80.8)$ & $125(100)$ & $96(76.8)$ & $73(58.4)$ & $118(94.4)$ \\
\hline & & $\mathbf{R}$ & $14(9.2)$ & $32(25.6)$ & $51(40.8)$ & $24(19.2)$ & 0 & $29(23.2)$ & $52(41.6)$ & $7(5.6)$ \\
\hline
\end{tabular}

Key: $\mathrm{R}=$ resistant $\mathrm{S}=$ sensitive, $\mathrm{RXN}=$ reaction, $\mathrm{CX}=$ cefoxitin, $\mathrm{CIP}=$ ciprofloxacin, $\mathrm{COT}=$ co-trimoxazole, $\mathrm{GEN}=$ gentamicin, $\mathrm{VA}=$ vancomycin, $\mathrm{P}=$ penicillin $\mathrm{G}, \mathrm{E}=$ erythromycin, $\mathrm{TE}$ = tetracycline.

TABLE 4: Clindamycin resistance among MRSA and MSSA strains.

\begin{tabular}{|c|c|c|c|c|c|c|c|c|}
\hline \multirow{2}{*}{$\begin{array}{l}\text { Susceptibility } \\
\text { pattern } \\
\text { (phenotypes) }\end{array}$} & \multirow[b]{2}{*}{$\mathrm{E}$} & \multirow[b]{2}{*}{$\mathrm{CD}$} & \multirow[b]{2}{*}{$D$-test } & \multicolumn{2}{|c|}{ MSSA } & \multicolumn{2}{|c|}{ MRSA } & \multirow{2}{*}{$\begin{array}{l}\text { Total S. aureus } \\
\quad(n=125) \\
(\text { no. }(\%))\end{array}$} \\
\hline & & & & $\begin{array}{c}\text { HIV-infected } \\
(n=69)(\text { no. }(\%))\end{array}$ & $\begin{array}{c}\text { Non-HIV } \\
(n=42)(\text { no. }(\%))\end{array}$ & $\begin{array}{c}\text { HIV-infected } \\
(n=11)(\text { no. }(\%))\end{array}$ & $\begin{array}{c}\text { Non-HIV } \\
(n=3)(\text { no. }(\%))\end{array}$ & \\
\hline $\begin{array}{l}\text { Inducible } \\
\text { MLS }_{\mathrm{B}}-\left(\mathrm{iMLS}_{\mathrm{B}}\right)\end{array}$ & $\mathrm{R}$ & $S$ & Positive & $17(24.6)$ & $4(9.5)$ & 7 (63.6) & $2(66.7)$ & $30(24)$ \\
\hline $\begin{array}{l}\text { Constitutive } \\
\mathrm{MLS}_{\mathrm{B}}-\left(\mathrm{cMLS}_{\mathrm{B}}\right)\end{array}$ & $\mathrm{R}$ & $\mathrm{R}$ & Negative & $11(15.9)$ & 5 (11.9) & $3(27.3)$ & 0 & $19(15.2)$ \\
\hline $\mathrm{MS}_{\mathrm{B}}$ & $\mathrm{R}$ & S & Negative & $21(30.4)$ & $11(26.2)$ & $1(9.1)$ & $1(33.3)$ & $34(27.2)$ \\
\hline Susceptible & S & S & Negative & $20(28.9)$ & $22(52.4)$ & 0 & 0 & $42(33.6)$ \\
\hline
\end{tabular}

Key: $\mathrm{R}=$ resistant, $\mathrm{S}=$ sensitive, $\mathrm{E}=$ erythromycin, $\mathrm{CD}$ = clindamycin, $\mathrm{MS}_{\mathrm{B}}=$ macrolide-streptogramin $\mathrm{B}, \mathrm{MLS}_{\mathrm{B}}=$ macrolide-lincosamide-streptogramin $\mathrm{B}$.

TABLE 5: Nasal colonization of $S$. aureus with respect to risk factors.

\begin{tabular}{lccc}
\hline $\begin{array}{l}\text { Environmental } \\
\text { parameters }\end{array}$ & $\begin{array}{c}\text { S. aureus } \\
\text { carriers } \\
(n=125) \\
(\text { no. }(\%))\end{array}$ & $\begin{array}{c}\text { S. aureus } \\
\text { non-carriers } \\
(n=475) \\
(\text { no. }(\%))\end{array}$ & $\begin{array}{c}p \\
\text { value }\end{array}$ \\
\hline $\begin{array}{l}\text { Farm working/living } \\
\quad \text { Yes }\end{array}$ & $\begin{array}{l}4(33.6) \\
\quad \text { No }\end{array}$ & $306(64.4)$ & $<\mathbf{0 . 0 5}$ \\
$\quad$ Job conditions & $83(66.4)$ & $169(35.6)$ & \\
$\quad$ With close contact & $89(71.2)$ & $276(58.1)$ & $\mathbf{0 . 0 0 7 6}$ \\
$\quad$ Without close contact & $36(28.8)$ & $199(41.9)$ & \\
Current smoking habit & & & \\
$\quad$ Yes & $76(60.8)$ & $222(46.7)$ & $\mathbf{0 . 0 0 5 1}$ \\
$\quad$ No & $49(39.2)$ & $253(53.3)$ & \\
$\begin{array}{l}\text { Alcoholic/nonalcoholic } \\
\text { (current) }\end{array}$ & & & \\
$\quad \begin{array}{l}\text { Alcoholic } \\
\quad \text { Nonalcoholic }\end{array}$ & $69(55.2)$ & $234(49.3)$ & $\mathbf{0 . 3 1 9}$ \\
$\begin{array}{l}\text { Outpatient/inpatient } \\
\quad \text { Outpatient }\end{array}$ & $56(44.5)$ & $241(50.7)$ & \\
$\quad$ Inpatient & $56(44.8)$ & $279(58.7)$ & $\mathbf{0 . 0 0 5}$ \\
\hline
\end{tabular}

(64\%) than in male (36\%) patients from Iran [36]. Patients with close contact job were more MSSA carriers 71.2\% (89/ $125)$, as compared to $28.8 \%(36 / 125)$ patients who had no close contact and the result was statistically significant $(p=0.0076)$. Similar result was reported by Oliva et al. where $57 \%$ of people who had close contact job were MSSA carriers and $27.1 \%$ subjects were MSSA carriers who did not have human contact job [1]. This study shows higher rate of MSSA carriers among patients with current smoking habit $(p=0.0051)$ and patients who used to work or live in farm area $(p<0.05)$. Similarly, there were more MSSA carriers among inpatients (55.2\%) in comparison to out-patients $(44.8 \%)$. The association between type of patients (inpatients/out-patients) and MSSA carriers rate was statistically significant $(p=0.005)$. These results suggest that alcohol consumption, working/living in farm area and job with human contact environment positively contribute towards the spread of MSSA and MRSA. Moreover, hospitalized patients are at greater risk of having CA-MRSA colonization. Individual behavioral factors, environmental, social, HIV-host factors, and all these in united form play a positive role in S. aureus colonization and subsequent series of infection [30].

In this study, $94.4 \%(118 / 125)$ of $S$. aureus isolates were sensitive to tetracycline followed by cefoxitin $88.8 \%$ (111/ $125)$, whereas $9.2 \%(14 / 125)$ of isolates were resistant to cefoxitin. High rate of resistance $41.6 \%$ was observed against the antibiotic erythromycin followed by co-trimoxazole (40.8\%), ciprofloxacin (25.6\%) and penicillin G (23.2\%), respectively. There was $100 \%$ sensitivity to the antibiotic vancomycin. The rate of co-trimoxazole resistance was higher among S. aureus isolated from HIV-infected patients than non-HIV patients. Co-trimoxazole is being used as a major chemoprophylaxis agent for HIV-infected patients for the treatment of various bacterial infections. Increasing rate of antibiotic resistance among MRSA isolates may increase the burden of infections in the community and clinical setting especially in patients with HIV/AIDS [37]. Similar result was reported on antibiotic susceptibility pattern of 
S. aureus isolated from health care workers of western Nepal by Khanal et al. (2015) where $93.8 \%$ isolates were sensitive to tetracycline and $78.1 \%$ were sensitive to cefoxitin whereas $100 \%$ MRSA was penicillin resistant [31].

Among 125 S. aureus isolates, 24\% (30/125) were detected to have inducible clindamycin $\left(\mathrm{iMLS}_{\mathrm{B}}\right)$ resistance whereas high rate of $\mathrm{iMLS}_{\mathrm{B}}$ isolates were confirmed from MSSA 70\% $(21 / 30)$ than MRSA 30\% (9/30). Additionally, 15.2\% (19/125) constitutive $\mathrm{MLS}_{\mathrm{B}}$ and $27.2 \%$ (34/125) macrolidestreptogramin $\mathrm{B}\left(\mathrm{MS}_{\mathrm{B}}\right)$ resistance $S$. aureus were detected where $33.6 \%(42 / 125)$ of $S$. aureus isolates were clindamycin sensitive. None of the MRSA isolates were clindamycin sensitive. The results are not consistent with the findings reported by Prabhu et al. (2011) where $37.52 \%$ and $16.66 \%$ of $S$. aureus isolates from non-HIV patients were confirmed as $\mathrm{iMLS}_{\mathrm{B}}$ and $\mathrm{CMLS}_{\mathrm{B}}$ resistance, respectively [38]. The findings of this study show that D-test should be performed in routine antibiotic susceptibility test which could guide the appropriate treatment options during the infection caused by $S$. aureus and MRSA. The rate of $\mathrm{MLLS}_{\mathrm{B}}$ resistance of this study is higher as compared to previous reports by Ansari et al. and Adhikari et al. where they have reported 12.4\% [39] and 10\% [40] of inducible clindamycin-resistant $S$. aureus, respectively. This variation could be due to different clinical settings, type of patients, environment, S. aureus with different susceptibility patterns, and behavioral factors of patients [41]. Clindamycin is a drug of choice for the treatment of $S$. aureus infections but constitutive or inducible resistance to this antibiotic has been a major cause of treatment failure. Both MRSA and inducible clindamycin resistance $S$. aureus are serious challenge to health care management of Nepal especially in immunosuppressed groups like patients with HIV/ AIDS, tuberculosis, renal transplant, cancer, diabetes, liver cirrhosis, malignancy, and chronic cardiovascular diseases.

High prevalence of nasal colonization along with increased rate of antibiotic resistance in $S$. aureus has posed a great threat to public health of the country and beyond. Compromised immune system and other risk factors including behavioral characters of HIV patients have led to emergence of MRSA which ultimately results in increase burden of community and hospital acquired infections. Increasing treatment failure due to high level of antibiotic resistance of $S$. aureus has expanded the burden of diseases in developing countries. Routine surveillance, proper monitoring of antibiotic resistance pattern and implementation of control strategies to prevent circulation of $S$. aureus strains in both clinical and hospital settings along with early detection of pathogenic MRSA isolates is very crucial for the reduction of morbidity and mortality due to diverse forms of $S$. aureus infections. Hence, routine screening, investigation of $S$. aureus nasal carriage particularly in immune deficient individuals, monitoring of their antibiotic susceptibility and control of $S$. aureus transmission are preventive me2asures for proper management of infections due to $S$. aureus.

\section{Conclusion}

The findings of this study show high rates of $S$. aureus colonization (mainly MRSA) and subsequent infections among
HIV-infected patients. Smoking and alcohol drinking habits pose an individual at higher risk of $S$. aureus infection. Inducible clindamycin resistance $\left(\mathrm{iMLS}_{\mathrm{B}}\right) \mathrm{S}$. aureus in $\mathrm{HIV}$ patients is increasing. D-test for the detection of iMLS $_{B}$ can be included as a screening test in routine laboratory investigation. Special attention is urgent to keep transmission and emergence of MRSA strains under control in people living with HIV/AIDS. S. aureus isolates showed high frequency of sensitive to tetracycline but we should take in account geographic differences in AST pattern when selecting antibiotic. Control strategies and interventions are very crucial to stop the spread to this "superbug" beyond the border.

\section{Limitations}

We cannot reveal the exact figure of $S$. aureus colonization without investigating swabs of other probable body sites like groin, pharynx, axillae, and anus. Furthermore, we could not collect the other epidemiological risk factors which could directly affect the MSSA and MRSA colonization and infection pattern. We could not perform/provide the CD4 count data to correlate with the immune status of HIV infected individuals too. Due to limitation of laboratory resources we could not run molecular procedures for confirmation of mecA gene in MRSA isolates. Further investigation in molecular level with collection of broad risk factors of large number of participants from different parts of the nation is required to generalize the result.

\section{Abbreviations}

BA: Blood agar

CA: Chocolate agar

CDC: $\quad$ Centers for Disease Control and Prevention

MA: Mac-Conkey agar

MHA: Mueller Hinton agar

MDR: Multidrug resistance

MRSA: Methicillin-resistant S. aureus

CLSI: Clinical Laboratory Standards Institute

HIV: Human immunodeficiency virus

AIDS: Acquired immunodeficiency syndrome

ART: Antiretroviral therapy

AST: Antibiotic susceptibility testing

SCCmec: Staphylococcal cassettes chromosome

SOP: $\quad$ Standard operating procedure

PVL: $\quad$ Panton-Valentine Leukocidin

SSTI: Skin-and-soft tissue infection

NPHL: National Public Health Laboratory

WHO: World Health Organization

VRSA: Vancomycin-resistant S. aureus.

\section{Data Availability}

The data used to support the findings of this study are included within the article.

\section{Ethical Approval}

Letter of ethical approval for this study was obtained from Nepal Health Research Council with Reg. No. 368/2016, 
which can be presented in reasonable request, and the research proposal was approved by the Department of Microbiology, Tri-Chandra Multiple Campus, Kathmandu.

\section{Consent}

Well informed consent was obtained from all the patients in written form before sample collection.

\section{Conflicts of Interest}

The authors declare that there are no conflicts of interest regarding the publication of this article.

\section{Authors' Contributions}

$\mathrm{KN}$ is the primary author, who designed the study methodology, performed laboratory investigations, and prepared the initial manuscript. JA and PKS helped with the designing of the study, analyzing the results, editing and proofreading the article, managing necessary arrangements during laboratory investigations, and supervising the complete study. BR edited, proofread, analyzed the data, and formatted and revised the complete manuscript. NR, DS, BGC, and MRP edited and proofread the article, helped in data analysis, and revised the manuscript for submission. All authors approved the final manuscript before submission to the Canadian Journal of Infectious Diseases and Medical Microbiology.

\section{Acknowledgments}

The authors would like to acknowledge all the laboratory staffs of National Public Health Laboratory Teku, Kathmandu, for their valuable support during the study.

\section{References}

[1] A. Oliva, M. Lichtner, M. T. Mascellino et al., "Study of methicillin-resistant Staphylococcus aureus (MRSA) carriage in a population of HIV-negative migrants and HIV-infected patients attending an outpatient clinic in Rome," Annali di Igiene, Medicina Preventiva e di Comunità, vol. 25, no. 2, pp. 99-107, 2013.

[2] S. L. Yousef, S. Y. Mahmoud, and M. T. Eihab, "Prevalence of methicillin-resistant Staphylococcus aureus in Saudi Arabia: systemic review and meta-analysis," African Journal of Clinical and experimental Microbiology, vol. 14, no. 3, pp. 146-154, 2013.

[3] F. R. DeLeo, M. Otto, B. N. Kreiswirth, and H. F. Chambers, "Community-associated meticillin-resistant Staphylococcus aureus," The Lancet, vol. 375, no. 9725, pp. 1557-1568, 2010.

[4] J. Sheldon and D. Heinrichs, "The iron-regulated staphylococcal lipoproteins," Frontiers in Cellular and Infection Microbiology, vol. 2, p. 41, 2012.

[5] C. Pozzi, E. M. Waters, J. K. Rudkin et al., "Methicillin resistance alters the biofilm phenotype and attenuates virulence in Staphylococcus aureus device-associated infections," PLoS Pathogens, vol. 8, no. 4, article e1002626, 2012.

[6] V. C. Pereira, D. F. M. Riboli, and M. de Lourdes Ribeiro de Souza, "Characterization of the clonal profile of MRSA isolated in neonatal and pediatric intensive care units of a University Hospital," Annals of
Clinical Microbiology and Antimicrobials, vol. 13, no. 1, p. 50, 2014.

[7] R. M. Klevens, A. M. Melissa, J. Nadle et al., "Invasive methicillin-resistant Staphylococcus aureus infections in the United States," JAMA, vol. 298, no. 15, pp. 1763-1771, 2007.

[8] N. Safdar and E. A. Bradley, "The risk of infection after nasal colonization with Staphylococcus aureus," The American Journal of Medicine, vol. 121, no. 4, pp. 310-315, 2008.

[9] K. J. Popovich, K. Y. Smith, T. Khawcharoenporn et al., "Community-associated methicillin-resistant Staphylococcus aureus colonization in high-risk groups of HIV-infected patients," Clinical Infectious Diseases, vol. 54, no. 9, pp. 1296-1303, 2012.

[10] A. I. Hidron, E. V. Kourbatova, J. S. Halvosa et al., "Risk factors for colonization with methicillin-resistant Staphylococcus aureus (MRSA) in patients admitted to an urban hospital: emergence of community-associated MRSA nasal carriage," Clinical Infectious Diseases, vol. 41, no. 2, pp. 159-166, 2005.

[11] A. Senthilkumar, S. Kumar, and J. N. Sheagren, "Increased incidence of Staphylococcus aureus bacteremia in hospitalized patients with acquired immunodeficiency syndrome," Clinical Infectious Diseases, vol. 33, no. 8, pp. 1412-1416, 2001.

[12] L. S. Parasa, L. C. A. Kumar, P. Sirisha et al., "Epidemiological survey of methicillin resistant Staphylococcus aureus in the community and hospital, Gannavaram, Andhra Pradesh, South India," Reviews in Infection, vol. 1, no. 2, pp. 117-123, 2010.

[13] W. E. Bischoff, M. L. Wallis, K. B. Tucker, B. A. Reboussin, and R. J. Sherertz, "Staphylococcus aureus nasal carriage in a student community prevalence, clonal relationships, and risk factors," Infection Control and Hospital Epidemiology, vol. 25, no. 6, pp. 485-491, 2004.

[14] J. Lamaro-Cardoso, H. De Lencastre, Andre Kipnis et al., "Molecular epidemiology and risk factors for nasal carriage of Staphylococcus aureus and methicillin-resistant S. aureus in infants attending day care centers in Brazil," Journal of Clinical Microbiology, vol. 47, no. 12, pp. 3991-3997, 2009.

[15] F. D. Lowy, A. E. Aiello, M. Bhat et al., "Staphylococcus aureus colonization and infection in New York State prisons," Journal of Infectious Diseases, vol. 196, no. 6, pp. 911-918, 2007.

[16] N. Dhuria, P. Devi, B. Devi, and S. Malhotra, "Prevalence and risk factors for methicillin-resistant Staphylococcus aureus colonization in anterior nares of HIV-positive individuals," udpecker Journal of Medical Sciences, vol. 2, pp. 26-29, 2013.

[17] A. C. Smith and M. A. Hussey, "Gram stain protocols," in ACM Microbelibrary-Laboratory Protocols, American Society for Microbiology, Washington, DC, USA, 2005.

[18] B. A. Forbes, D. E. Sahm, and A. S. Weissfeld, Bailey and Scott's Diagnostic Microbiology, International Edition, Mosby, Inc., New York, NY, USA, 12th edition, 2007.

[19] CLSI, Performance Standards for Antimicrobial Susceptibility Testing, Clinical and Laboratory Standards Institute, Wayne, PA, USA, 2011.

[20] CLSI, Performance Standards for Antimicrobial Susceptibility Testing; Twenty-Fifth Informational Supplement, CLSI Document M100-S25, Clinical and Laboratory Standards Institute, Wayne, PA, USA, 2015.

[21] R. E. Nelson, M. E. Evans, L. Simbartl et al., "Methicillinresistant Staphylococcus aureus colonization and pre-and post-hospital discharge infection risk," Clinical Infectious Diseases, 2018. 
[22] H. Ringberg, A. C. Petersson, M. Walder, and P. J. H. Johansson, "The throat: an important site for MRSA colonization," Scandinavian journal of infectious diseases, vol. 38, no. 10, pp. 888-893, 2006.

[23] W. M. Kyaw, L. K. Lee, W. C. Siong, A. C. L. Ping, B. Ang, and Y. S. Leo, "Prevalence of and risk factors for MRSA colonization in HIV-positive outpatients in Singapore," AIDS Research and Therapy, vol. 9, no. 1, p. 33, 2012.

[24] W. C. Mathews, J. C. Caperna, R. E. Barber et al., "Incidence of and risk factors for clinically significant methicillin-resistant Staphylococcus aureus infection in a cohort of HIV-infected adults," Journal of Acquired Immune Deficiency Syndromes, vol. 40, no. 2, pp. 155-160, 2005.

[25] K. H. Gonsu, S. L. Kouemo, M. Toukam, V. N. Ndze, and S. S. Koulla, "Nasal carriage of methicillin resistant Staphylococcus aureus and its antibiotic susceptibility pattern in adult hospitalized patients and medical staff in some hospitals in Cameroon," Journal of Microbiology and Antimicrobials, vol. 5, no. 3, pp. 29-33, 2013.

[26] L. A. F. Reinato, D. P. M. Pio, L. P. Lopes, F. M. V. Pereira, A. E. R. Lopes, and E. Gir, "Nasal colonization with Staphylococcus aureus in individuals with HIV/AIDS attended in a Brazilian Teaching Hospital," Revista Latino-Americana de Enfermagem, vol. 21, no. 6, pp. 1235-1239, 2013.

[27] A. Habeeb, N. R. Hussein, M. S. Assafi, and S. A. Al-Dabbagh, "Methicillin resistant Staphylococcus aureus nasal colonization among secondary school students at Duhok City-Iraq," Journal of Microbiology and Infectious Diseases, vol. 4, no. 2, pp. 59-63, 2014.

[28] C.-S. Chen, C.-Y. Chen, and Y.-C. Huang, "Nasal carriage rate and molecular epidemiology of methicillin-resistant Staphylococcus aureus among medical students at a Taiwanese university," International Journal of Infectious Diseases, vol. 16, no. 11, pp. e799-e803, 2012.

[29] N. Akhtar, "Staphylococcal nasal carriage of health care workers," Journal of College of Physicians and Surgeons Pakistan, vol. 20, no. 7, pp. 439-443, 2010.

[30] A. I. Hidron, K. Russell, A. Moanna, and D. Rimland, "Methicillin-resistant Staphylococcus aureus in HIV-infected patients," Infection and Drug Resistance, vol. 3, p. 73, 2010.

[31] R. Khanal, S. Prakash, P. Lamichhane, A. Lamsal, S. Upadhaya, and V. Kumar Pahwa, "Nasal carriage of methicillin resistant Staphylococcus aureus among health care workers at a Tertiary Care Hospital in Western Nepal," Antimicrobial Resistance and Infection Control, vol. 4, no. 1, p. 39, 2015.

[32] F. R. DeLeo, B. A. Diep, and M. Otto, "Host defense and pathogenesis in Staphylococcus aureus infections," Infectious Disease Clinics of North America, vol. 23, no. 1, pp. 17-34, 2009.

[33] C. Cianflone, F. Nancy, A. A. Burgi, and B. R. Hale, "Increasing rates of community-acquired methicillin-resistant Staphylococcus aureus infections among HIV-infected persons," International Journal of STD and AIDS, vol. 18, no. 8, pp. 521-526, 2007.

[34] B. A. Diep, S. R. Gill, R. F. Chang et al., "Complete genome sequence of USA300, an epidemic clone of communityacquired meticillin-resistant Staphylococcus aureus," The Lancet, vol. 367, no. 9512, pp. 731-739, 2006.

[35] J. D. Szumowski, K. M. Wener, H. S. Gold et al., "Methicillinresistant Staphylococcus aureus colonization, behavioral risk factors, and skin and soft-tissue infection at an ambulatory clinic serving a large population of HIV-infected men who have sex with men," Clinical Infectious Diseases, vol. 49, no. 1, pp. 118-121, 2009.
[36] N. Fard-Mousavi, G. Mosayebi, A. Amouzandeh-Nobaveh, A. Japouni-Nejad, and E. Ghaznavi-Rad, "The dynamic of Staphylococcus aureus nasal carriage in central Iran," Jundishapur Journal of Microbiology, vol. 8, no. 7, 2015.

[37] M. J. A. Reid, A. P. Steenhoff, N. Mannathoko et al., "Staphylococcus aureus nasal colonization among HIVinfected adults in Botswana: prevalence and risk factors," AIDS Care, vol. 29, no. 8, pp. 961-965, 2017.

[38] K. Prabhu, S. Rao, and V. Rao, "Inducible clindamycin resistance in Staphylococcus aureus isolated from clinical samples," Journal of Laboratory Physicians, vol. 3, no. 1, p. 25, 2011.

[39] S. Ansari, H. P. Nepal, R. Gautam et al., "Threat of drug resistant Staphylococcus aureus to health in Nepal," BMC infectious diseases, vol. 14, no. 1, p. 157, 2014.

[40] R. Adhikari, N. D. Pant, S. Neupane et al., "Detection of methicillin resistant Staphylococcus aureus and determination of minimum inhibitory concentration of vancomycin for Staphylococcus aureus isolated from pus/wound swab samples of the patients attending a tertiary care Hospital in Kathmandu, Nepal," Canadian Journal of Infectious Diseases and Medical Microbiology, vol. 2017, Article ID 2191532, 6 pages, 2017.

[41] B. Shrestha, B. M. Pokhrel, and T. M. Mohapatra, "Phenotypic characterization of nosocomial isolates of Staphylococcus aureus with reference to MRSA," Journal of Infection in Developing Countries, vol. 3, no. 7, pp. 554-560, 2009. 


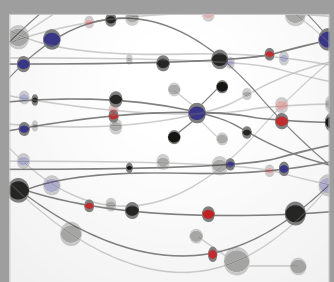

The Scientific World Journal
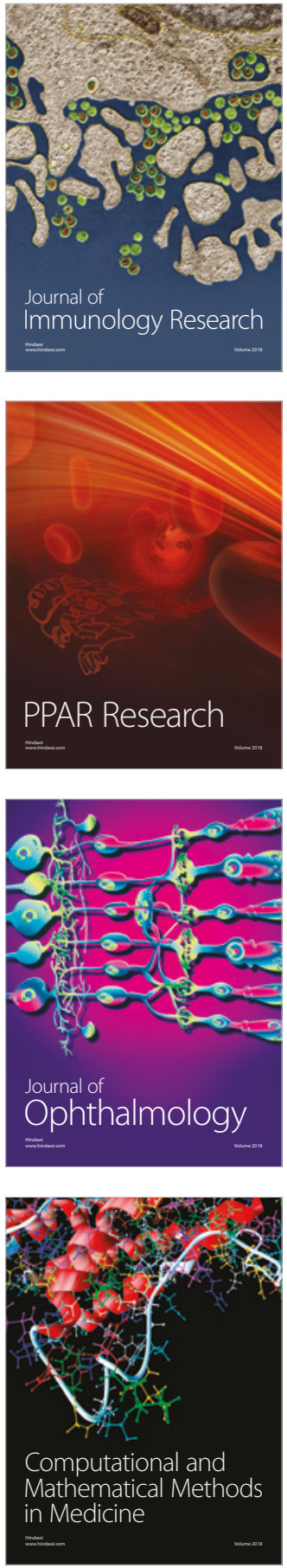

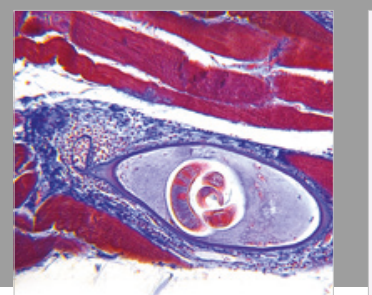

Gastroenterology Research and Practice

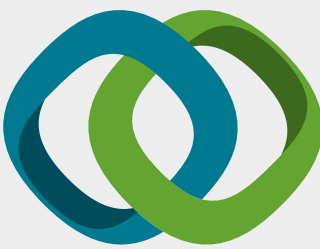

\section{Hindawi}

Submit your manuscripts at

www.hindawi.com
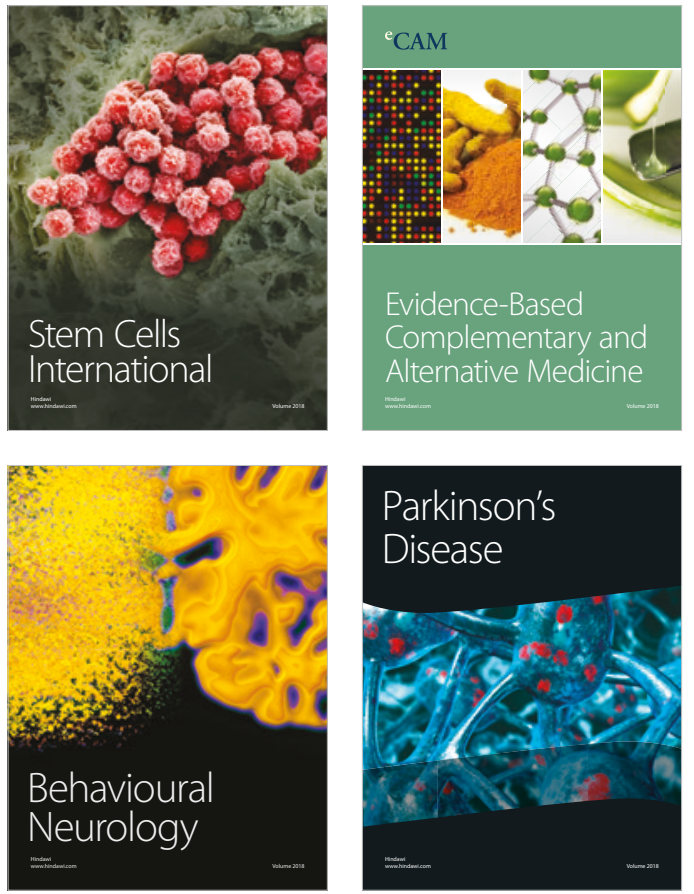

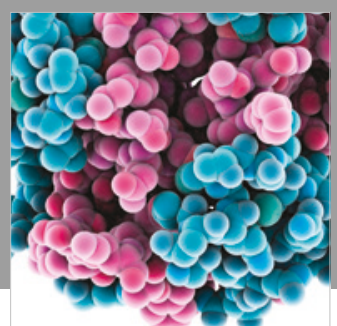

ournal of

Diabetes Research

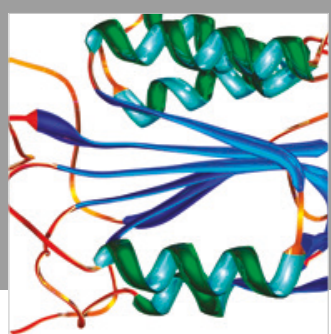

Disease Markers
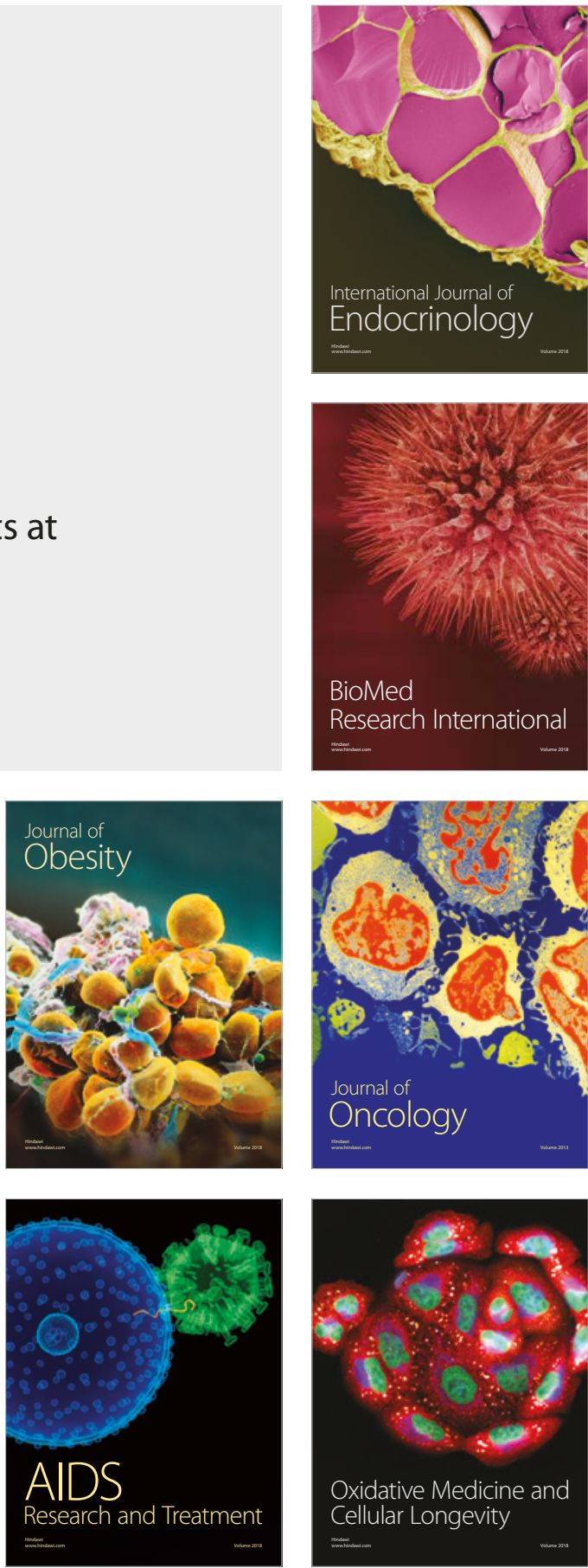